\title{
Maxillofacial Radiology 175
}

\author{
SADJ October 2019, Vol. 74 No. 9 p508
}

CJ Nortjé

Below are a clinical picture and radiographs of two patients presenting with recurring draining abscesses affecting the mandible. Intensive antibiotic treatment achieved no noticeable improvement. Discuss the important radiological features and what is your diagnosis?
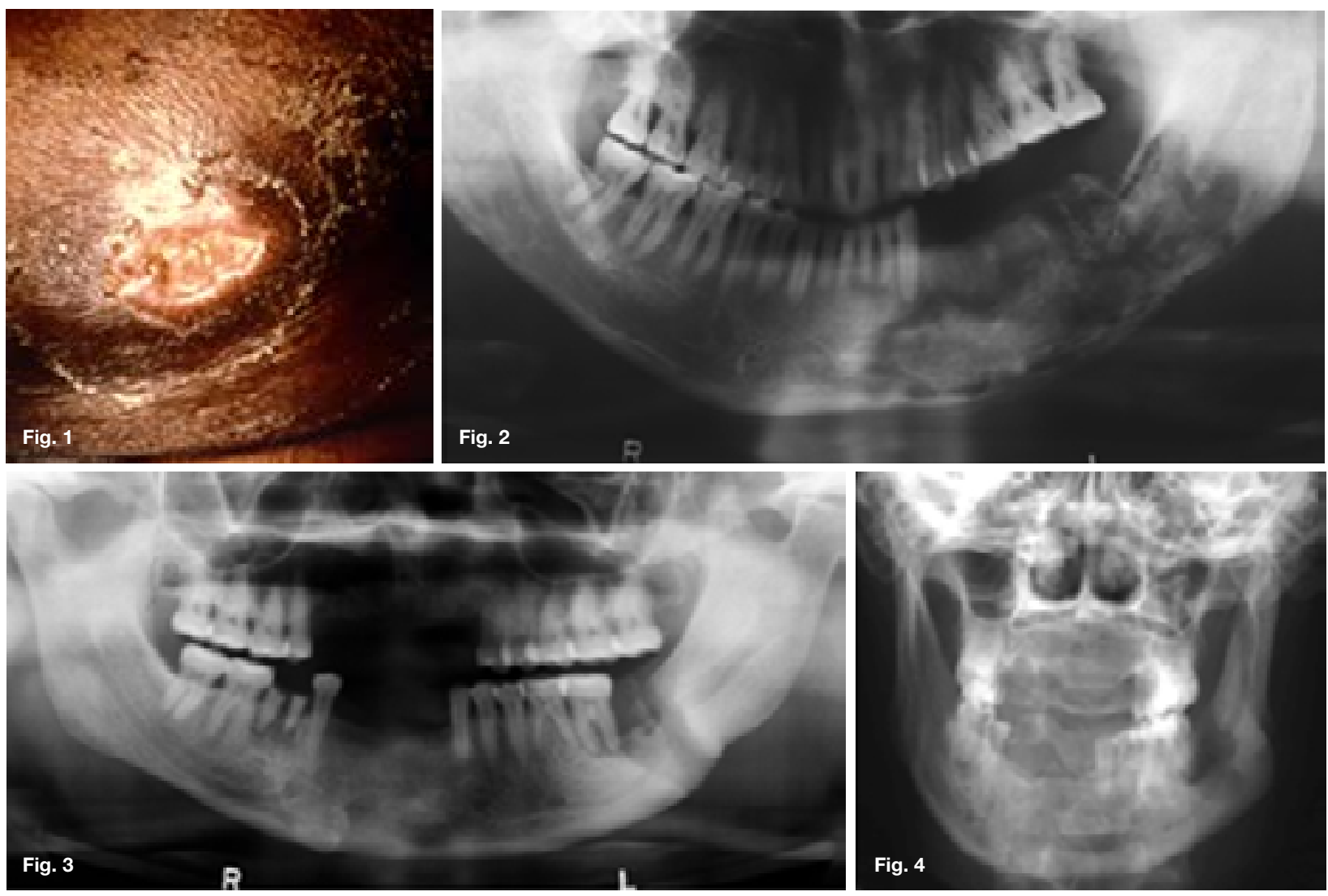

INTERPRETATION

The upper two figures show a case in which there are facial scars of a recurring infection while the pantomograph shows ill-defined multilocular radiolucencies with enlargement of the trabecular spaces, suggestive of the presence of a chronic osteomyelitis affecting the hemiandible on the left side. There are also features demonstrating the formation of sequestra. The case shown in the lower figures started after an assault which had caused a fracture at the angle of the left mandible. A tooth in line of the fracture was removed and since then a draining sinus had developed which showed signs of healing after the removal of sequestrum. In both cases a diagnosis of chronic actionomycotic osteomyelitis of the mandible was made, a rare condition found in $10 \%$ of all cervicofacial actinomycosis cases. When it occurs, the radiographic changes are not characteristic. Actionomycosis is a specific infectious disease, primarily caused by the organism Actinomycis Israëli. It affects men twice as frequently as women and

Christoffel J Nortjé: $B C h D, P h D, A B O M R, D S c$. Faculty of Dentistry, University of the Western Cape.

ORCID Number: 0000-0002-9717-5514

Email: cnortje@uwc.ac.za is most often encountered in the 20-50 year age groups. The cervicofacial type of actinomycosis is by far the most common, with pulmonary and abdominal actinomycosis occurring less frequently. However, because of its variable clinical manifestations and difficulty in isolating the causative agent, the diagnosis is often delayed. The actinomycotic infection is not a pure infection; it probably is a result of a synergism between microbes of the ray fungus group and other anaerobic microbes. Clinically, the infection has a prolonged course. The first sign is the appearance of a red to dark blue, nodular infiltration, often located within the skin of the submandibular area or angle of the jaw. Untreated, the infection spreads along anatomical planes, producing a hard infiltrate with simultaneous occurrence of draining abscesses. The exudate may contain small yellow granules, termed "sulphur granules", which consist of colonies of actinomyces which tends to be diagnostic. Enlargement of regional lymphnodes is seldom observed and the general status of the patients is unimpaired.

\section{Reference}

1. Pindborg, JJ and Hjorting-Hanson, E: Atlas of Diseases of the Jaws, WB Saunders, 1974, p20. 